\title{
Ebola drug trial is to start next month
}

\author{
Anne Gulland
}

London

The first clinical trial of drugs to treat Ebola virus disease is set to launch at the beginning of November, with first results available by the end of the year, one of the trial investigators has told The BMJ.

Researchers are currently visiting treatment centres run by the charity Médecins Sans Frontières and other organisations in the three worst affected countries, Sierra Leone, Guinea, and Liberia, to assess whether they might be able to support a clinical trial, alongside their crucial work of providing care and treatment.

This is the first time a clinical trial will be carried out during a disease outbreak. The partnership behind the trial, which includes Oxford University, the International Severe Acute Respiratory and Emerging Infections Consortium, the Global Health Network, the Institut Pasteur, and the Institut Pasteur de Dakar, is preparing the groundwork at record speed, having been awarded a $£ 3.2 \mathrm{~m}(€ 4 \mathrm{~m} ; \$ 5.1 \mathrm{~m})$ grant by the Wellcome Trust at the end of September.

"Generally, it takes about 18 months to set up a clinical trial, but we're doing everything in parallel," said Trudie Lang, one of the co-investigators and professor of global health research at Oxford University. "It's incredible," she added. "Everyone is being so supportive and working together on this-we're going to change the clinical trial paradigm if we can get this done."

Lang could not say which drug would be tested first, as the final agreement had not yet been made with the drug company, but the first trial would be one of several testing a range of treatment candidates.

Lang underlined the importance of working in partnership and alongside researchers from the affected countries. The consortium will use local investigators and research staff where possible. She added that about 50 African research nurses had already volunteered to help run the trials.

"We will work with local health workers and the charities that are providing the current treatment and care, but they're not trained to conduct research, so we also need to build capacity and provide this training," Lang said. "To do this we need to bring in people who know how to create that different environment of being a trial. That's why we want to bring in African research nurses to support and work with the medical teams to conduct the key trial requirements, such as observing that the drug has been given and assess whether there are any side effects."
Meanwhile, the World Health Organization has said that the first vaccines against the Ebola virus may be in use among frontline workers in west Africa by the beginning of next year. The two "front running" vaccines are cAd3-ZEBOV, a chimpanzee derived adenovirus vaccine developed by GlaxoSmithKline in conjunction with the US National Institute of Allergies and Infectious Diseases, and rVSV-ZEBOV, developed by the Public Health Agency of Canada and now licensed to a US company called New Link.

The chimpanzee vaccine is in clinical trials in the United States, the United Kingdom, and Mali and will enter clinical trials in Switzerland at the beginning of next month. The second vaccine is in trials in the US and will also be tested in volunteers in Germany, Switzerland, Gabon, and Kenya.

"These trials will incorporate the largest number of volunteers and will be very important in terms of determining both the safety and immunogenicity of the vaccines," said WHO assistant director general Marie-Paule Kieny at a press conference in Geneva earlier this week.

The European phase of the trials will start in the coming weeks, and the initial safety and immunogenicity results will be available at the end of the year, said Kieny. Then the vaccines will begin testing in January among health workers and burial teams.

Kieny emphasised that the initial vaccination campaign in west Africa would be small, adding, "There's still a possibility it will fail, but everybody is putting things in order for being able to move to west Africa in January."

Kieny admitted that WHO and GlaxoSmithKline had decided not to fast track the vaccine in March this year, as WHO thought that the best way to tackle the Ebola outbreak was by the previously successful methods of contact tracing and isolation of patients.

"The vaccine had never been tested in any human beings, and the normal development of a vaccine takes many years," she said. "There was absolutely no belief that a vaccine could be brought online in enough time."

Partnerships are also being set up in the affected countries to test treatments that use convalescent blood and serum, the most advanced of which is in Liberia.

Cite this as: BMJ 2014;349:96436

๑ BMJ Publishing Group Ltd 2014 